Indian J. Anim. HIth. (2019), 58(2) : 245-248

DOI: https://doi.org/10.36062/ijah.58.2.2019.245-248

News \& Views

\title{
INVENTORS OF CELLULAR OXYGEN SENSOR RECOGNIZED AS NOBEL PRIZE WINNERS
}

\author{
TAPAS GOSWAMI \\ Ex-Principal Scientist \\ Immunology Section, Indian Veterinary Research Institute \\ Izatnagar-243 122, Utter Pradesh, India
}

\section{Introduction}

Animals need oxygen for the conversion of food into useful energy. On the contrary origin of animal from low oxygen world has been scientifically proved. In minimal presence of oxygen, lower invertebrates such as sponges (under phylum porifera) can survive but not vertebrates (Knoll and Sperling, 2014). If this is the case, then there must be something more by which human being and animals withstand low oxygen availability. The term "hypoxia" indicates deficiency of gaseous oxygen reaching the tissues, cells or organelles of the body. Oxygen requirement reflects size of the animal and metabolic demand of the cells within tissue. There is high oxygen demand to respiring tissues for its maintenance and it escalates during exercise. Hypoxia may be categorized as ambient hypoxia, anemic hypoxia, stagnant hypoxia and histotoxic hypoxia. One thing common for the above is limited oxygen availability rather than anoxia (complete absence of oxygen). The present discussion is intended to elaborate oxygen sensor mechanism of cells by which it can sense the available oxygen level so that cellular functions continue. Recently during $7^{\text {th }}$ October 2019 , the subject of molecular mechanism of oxygen sensor has gone in favour of Physiology or Medicine Nobel Prize winners. The criticality of the oxygen sensor mechanism has been unfolded by three scientists namely William Kaelin, Jr. Sir Peter Ratcliffe and Gregg Semenza. Within tissues, oxygen levels fluctuate dynamically, therefore cells are equipped with mechanism that can sense oxygen tension to adjust metabolism. Oxygen sensing ability of cells is crucial for fetal development, and equally important for tumor growth. The significance of oxygen in cell physiology has long been documented. During evolution, certain organs have behaved as typical $\mathrm{O}_{2}$ sensing tissues, such as the carotid artery, pulmonary artery, adrenal chromaffin cells and mitochondria (Aragones et al., 2009).

In the present report, description of these classical oxygen sensors have been excluded rather effort has been made to elaborate how cells sense oxygen levels fluctuation by gene switching mechanisms and maintain its physiological function from the available published literature. 


\section{Oxygen deprivation situation}

Before going detail of the gene regulation mechanism to sense the oxygen availability inside a cell, it is imperative to simplify the subject for the readers. Physiological adaptation of animals to low oxygen level in high altitude can be grouped into respiratory, circulatory, and hematological adaptation. All animals require molecular oxygen for oxidative phosphorylation to catabolize the sugar molecules to generate energy in the form of ATP. Specialized organs such as lungs, heart, blood and vascular system are developed in higher vertebrates to facilitate oxygen delivery to the cells and tissues. During exercise or when blood flow is interrupted there is deprivation of oxygen to tissues. Animal cells are equipped to adjust their metabolic rates to sustain variations in oxygen levels. The situation arises during (i) tissue injury, which requires tissue remodeling by reconstructing vasculature (angiogenesis), (ii) during exercise by increasing ventilator response. Further, when animals are located at high altitude where partial pressure of oxygen is low, there is a need to adapt its metabolic process sensing the low oxygen level. In healthy conditions, not all cells in our body are exposed to the same level of $\mathrm{O}_{2}$. Differences in the partial pressure of oxygen $\left(\mathrm{pO}_{2}\right)$ in distinct anatomical sites and physiological conditions are observed. In most tissues, the $\mathrm{pO}_{2}$ varies between 20 to $45 \mathrm{~mm} \mathrm{Hg}$, but in kidney medulla, bone marrow or intrauterine fetal compartment, the $\mathrm{pO}_{2}$ is sufficiently low, ranging between 10 to $25 \mathrm{~mm}$ $\mathrm{Hg}$. In solid tumor certain regions are exposed to extreme hypoxic state $(<0.1 \mathrm{mmHg})$, near to anoxia (Aragones et al., 2009). All mammalian cells, and not just specialized chemoreceptors, respond to changes in oxygen level. Hypoxia leads to major human diseases such as myocardial ischemia, stroke and cancer.

\section{Erythropoietin playing at center forward}

Once anemic conditions in pet become unmanageable by haematinics, the need of erythtopoietin has been realized. The erythropoietin (EPO), a hormone released from kidney induces erythropoiesis. Even at normal hypoxia there is rise in level of EPO. When animals are exposed to low ambient oxygen at high altitude, variation of oxygen level in blood is sensed and EPO is secreted from kidney. Erythropoietin activates red blood cell (RBC) synthesis from reserved stem cells of bone marrow. This results in higher RBC count to adapt lower oxygen stress. RBC contains haemoglobin, which ferries oxygen throughout the body. So making more RBC means more oxygen availability to cells and tissues. Historically more than century ago French physiologist Paul Bert in 1882 already demonstrated red blood cell count increased on exposure to high altitude (Bert, 1882). Therefore, the importance of physiological adaptation to low oxygen situation was already known, but how this process was controlled by $\mathrm{O}_{2}$ remained a mystery. Specific DNA segment located adjacent to the EPO gene was found to be the key mediator to exhibit its response to hypoxia. From the above discussion one can predict that during hypoxia some intrinsic factors may be involved which act as inducer for erythropoiesis. Possibility is that these factors may not be extraneous agent rather biological agent (gene product/ protein) that can activate or shut down few or more gene in a cyclic manner. The variation in oxygen level triggers more than 300 genes for the survival of animals. 


\section{Hypoxia inducible factor center of orchestra}

The molecular complexity to the response of oxygen flux is controlled by a transcription factor. This transcription factor is known as hypoxia inducible factor (HIF), reported by Gregg Semenza and his group. HIF is a group of proteins that turns on the activity of the EPO genes to synthesize EPO and other proteins (Wang and Semenza, 1995). HIF was initially identified in erythropoietin producing hepatoma cells and kidney cells. Subsequently the presence of EPO gene was confirmed in those species lacking erythropoietin. Existence of EPO gene has been confirmed even those species lacking red blood cells. HIF is composed of structurally two different proteins having $\alpha$ and $\beta$ subunits. The subunit HIF- $\alpha$ is involved in response to hypoxia but not $B$ subunit. At low oxygen level HIF- $\alpha$ concentration is high enough, so that it can bind to EPO gene and thus regulate EPO gene function. When oxygen level is normal the cells contain very little HIF- $\alpha$. The degradation of HIF- $\alpha$ is oxygen dependant and regulated by another gene product.

\section{Chemical modification as switch mechanism}

Upstream (5' prime region of the DNA strand) to the HIF coding region, another gene segment, called VHL (von Hippel-Lindau) gene, is located. VHL disease is a highly angiogenic tumor growth in families with VHL mutation that is characterized by highly angiogenic tumor and overproduction of erythropoietin. HippelLindau tumor suppressor protein from VHL gene inhibits tumor growth in normal cell, once mutated the tumor grows rapidly (Ratcliffe,
2013). VHL protein normally cleaves $\alpha$ subunit of HIF, so that it mediates oxygen dependant instability of HIF. The HIF- $\alpha$ subunits are rapidly degraded by the proteasome (protein degrading organels of a cell) under normal condition of oxygen level, but are stabilized during hypoxia. Hypoxia leads to synthesis of HIF. Accumulated HIF induces production of EPO from kidney to adjust metabolism to $\mathrm{O}_{2}$ supply. The interaction of VHL with $\alpha$ subunits of HIF is mediated in presence of iron and oxygen. This has indicated oxygen dependant chemical modification of HIF- $\alpha$ subunits that facilitate VHL binding resulting enzymatic (prolyl hydroxylases) cleavage of HIF degradation. Two independent reports published from Ratcliffe and Willim Kaelin lab at distant location advocated for hydroxylation of proline residues in HIF- $\alpha$ domain. Hydroxylation enhances the binding affinity for VHL- complex (Ivan et al., 2001; Jaakkola et al., 2001). The binding mechanism stands valid because hydroxylation of proline residues needs presence of oxygen whereas in absence of oxygen hydroxylation can't proceed. If we simplify the above mechanism, it states that when oxygen is low, hydroxyl groups don't get added and VHL ignores to bind with HIF. This allows HIF to switch on EPO gene to synthesize EPO and other proteins needed to survive low oxygen stress.

\section{Benefit to mankind}

One of the members of the Nobel assembly Randall Johnson described the work as "Text Book Discovery" and said students would start learning at the most basic levels of biology education.

The significant role of HIF system has been 
confirmed in anemia, cancer, heart attack, stroke and other disorders. If central part of tumor mass is exposed to extreme hypoxia, then how tumor grows, is a matter of concern. Diffusion of oxygen is limited only to an extent of few millimeters away from blood capillaries before the cells around it use up the oxygen. In this adverse situation with tumor growth beyond 1 millimeter depth, there is absolute need to generate new blood vessel. There must be mechanism which operates under hypoxic conditions by which tumor grow rapidly. The present research finding can be applied to close that window so that tumor growth is arrested. Scientific effort has been initiated to arrest the

\section{REFERENCES}

Aragones J, Fraisl P, Baes M and Carmeliet $\mathrm{P}, 2009$. Oxygen sensor at the cross road of metabolism. Cell Metabol, 9: 11-22

Bert P, 1882. Sur la richesse en hemoglobine du sang des animaux vivant sur les haut lieux. Comptes rendus del'Académie des Sciences, 94: 805-807

Ivan M, Kondo K, Yang H, Kim W, Valiando J et al., 2001. HIF alpha targeted for VHLmediated destruction by proline hydroxylation: implications for $\mathrm{O}_{2}$ sensing. Science, 292: 464-468

Jaakkola P, Mole DR, Tian YM, Wilson MI, oxygen supply to the growing tumor so to suffocate cancer cells by switching off their HIF system. New approach has been taken up to limit damage from heart attacks or kidney disease by turning on low-oxygen response. Researchers are looking for a treatment regime to switch off the HIF so that cancer cells will be deprived of oxygen and may not grow further. Recent drug in the name of 'roxadustat', already marketed in China, is used to block the enzyme that normally breakdown HIF. For clinical treatment of anemia due to chronic kidney disease, 'roxadustat' is used. Blocking the enzyme keeps the switch on to increase production of EPO.

Gielbert J et al., 2001. Targeting of HIFalpha to the von Hippel-Lindau ubiquitylation complex by $\mathrm{O}_{2}$-regulated prolyl hydroxylation. Science, 292: 468-472

Knoll AH and Sperling EA, 2014. Oxygen and animals in earth history. PNAS, 111(11): 3907-3908

Ratcliffe PJ, 2013. Oxygen sensing and hypoxia signaling pathways in animals: The implication of physiology of cancer. J Physiol, 591(8): 2027-2042

Wang GL and Semenza GL, 1995. Purification and characterization of hypoxia-inducible factor 1. J Biol Chem, 270: 1230-1237 